\title{
Prothrombinex-VF use in warfarin reversal and other indications
}

\section{Paul C Kruger \\ MB BS, \\ Haematology \\ Advanced Trainee \\ Annette S Le Viellez \\ BSc(MedSci), Scientist in Charge, Transfusion Medicine ${ }^{2}$ \\ Richard P Herrmann MB BS, FRCPA, FRACP Haematologist ${ }^{2}$ \\ 1 Department of Haematology, Fremantle Hospital and Health Service, Fremantle, WA. \\ 2 Haematology Department, Royal Perth Hospital, Perth, WA. \\ Paul.Kruger@ health.wa.gov.au}

MJA 2012; 196: 462-465 doi: 10.5694/mjall.10797
. rothrombinex-VF powder for injection (PTX-VF; CSL Biotherapies, Melbourne, Vic) is a prothrombin complex concentrate (PCC). Each vial contains $500 \mathrm{IU}$ factor II, $500 \mathrm{IU}$ factor IX, $500 \mathrm{IU}$ factor $X$, 25 IU antithrombin III, 192 IU heparin sodium and $\leqslant 500 \mathrm{mg}$ human plasma proteins. PTX-VF is indicated in the treatment and perioperative prophylaxis of bleeding in acquired deficiency of coagulation factors caused by vitamin $\mathrm{K}$ antagonists (ie, warfarin), and the treatment and prophylaxis of bleeding in patients with congenital coagulation factor deficiency. However, clinical data on the efficacy of PTX-VF are limited and the optimal dose is yet to be determined. ${ }^{1-3}$ Locally, the Australasian Society of Thrombosis and Haemostasis Warfarin Reversal Consensus Group recommends 25-50 IU/kg of PTX-VF combined with fresh frozen plasma (FFP) or vitamin $\mathrm{K}^{4}{ }^{4}$ However, previous audits suggest that relatively low doses effectively reduce international normalised ratio (INR), even without FFP. ${ }^{5}$

This audit of PTX-VF use aimed to assess: the effect of lower $(<25 \mathrm{IU} / \mathrm{kg})$ versus higher doses $(25-50 \mathrm{IU} / \mathrm{kg})$ for warfarin reversal; the effect when combined with FFP for warfarin reversal; use for indications other than warfarin reversal; and safety profile.

\section{Methods}

Royal Perth Hospital (RPH) is Western Australia's largest hospital; it has 855 inpatient beds and its medical and surgical specialties include major trauma, burns, heart and lung transplantation, bone marrow allografting and interventional neuroradiology. PTX-VF is stored in the Transfusion Medicine Unit of the Haematology Department and dispensed after request from medical officers.

All patients prescribed PTX-VF at RPH from 1 November 2009 to 1 May 2010 were identified from the ULTRA (GE Medical Systems, Milwaukee, Wis, USA) computer database. A

\begin{abstract}
Objective: To assess the use of Prothrombinex-VF powder for injection (PTX-VF) at Royal Perth Hospital and analyse the efficacy and safety profile of PTX-VF.

Design, setting and patients: A prospective observational audit of PTX-VF use, conducted by reviewing medical records and laboratory and imaging results for all patients prescribed PTX-VF from 1 November 2009 to 1 May 2010.

Main outcome measures: Data on indication, diagnosis, comorbidities, dose of PTX-VF, fresh frozen plasma (FFP) and vitamin K, coagulation parameters before and after PTX-VF administration, and adverse effects.

Results: 334 vials of PTX-VF were administered to 84 patients over 107 prescriptions. Indications were warfarin reversal, intraoperative bleeding and coagulopathy (66, 20 and 21 prescriptions, respectively). PTX-VF with FFP was compared with PTX-VF alone for warfarin reversal and there was a significant decrease in international normalised ratio (INR) that was independent of group $(P<0.001)$. Lower doses of PTX-VF $(<25 \mathrm{IU} / \mathrm{kg})$ were compared with higher doses (25-50 IU/kg) for warfarin reversal and decrease in INR was significant, independent of group $(P=0.002)$. PTX-VF was administered for intraoperative bleeding in 18 patients who had not been treated with warfarin. No hypersensitivity reactions, thrombotic complications or worsening of disseminated intravascular coagulation occurred during 7-day follow-up.

Conclusion: For warfarin reversal, lower doses of PTX-VF $(<25 \mathrm{IU} / \mathrm{kg})$ and PTX-VF without FFP were effective. PTX-VF was also used in intraoperative bleeding and non-warfarin coagulopathy. No adverse events were associated with PTX-VF.
\end{abstract}

prescription was defined as administration of PTX-VF with blood tests measuring coagulation profile before and after. To enable observation of raw patterns of use, medical officers at $\mathrm{RPH}$ were not aware of the audit. Permission for the audit was given by the RPH Human Research Ethics Committee.

We collected data on: patient demographics (age, sex), medical history (weight, indication for PTX-VF, diagnosis and past medical history) and drug history (warfarin, aspirin and/or clopidogrel within 5 days of PTX-VF administration); prescription details (dose of PTX-VF, FFP, vitamin $\mathrm{K}$ and other haemostatic agents); results of laboratory investigations (INR, activated partial thromboplastin time [APTT], fibrinogen level and platelet counts before and after PTXVF administration); and adverse effects (hypersensitivity reactions, thrombosis and disseminated intravascular coagulation). Patients were followed up for 7 days after administration of PTX-VF, or until death if this occurred sooner, by review of medical records and results of laboratory and imaging tests.

\section{Statistical analysis}

Patients with multiple PTX-VF prescriptions could not be compared with those who had a single prescription (because of insufficient patient numbers), so a standard error adjustment for the correlation within the clusters of observations for patients with multiple prescriptions was included in all analyses. For continuous outcomes, bootstrapped linear regression was used; for dichotomous measures of vitamin $\mathrm{K}$ and FFP (administered or not), logistic regression was used. Regression models were used to test bivariate relationships to facilitate the standard error adjustment necessary for the clustered observations. Where possible, transformations to geometric mean were applied to produce normally distributed outcomes. Geometric means of PTX-VF doses were obtained by taking the antilog of the mean of the log transformed PTX-VF dose. For the change in INR over time, random-effects regression was performed on the inverse of the INR value, which was normally distributed. Mean INR values are backtransformed means of inverse INR values. 


\section{Results}

During the 6-month audit period, 334 vials of PTX-VF were prescribed to 84 patients. Sixty-nine patients had a single prescription, 12 patients had two prescriptions, and the three remaining patients had three, four and seven prescriptions respectively, thus totalling 107 prescriptions. Every vial of PTX-VF used during the audit period was accounted for. No patient had a documented history of coagulation factor deficiency. The mean time between PTX-VF administration and INR measurement after treatment was 4.5 hours.

Indications for PTX-VF fell into three groups: warfarin reversal, intraoperative bleeding, and coagulopathy (prolonged INR [greater than 1.5] not due to warfarin). Some patients had overlapping indications and multiple medical teams involved in their care, therefore the predominant indication was used for our analyses (Box 1). The numbers of vials prescribed by specialty and indication are shown in Box 2.

\section{Warfarin reversal}

PTX-VF was prescribed 66 times for warfarin reversal because of imminent surgery (33 prescriptions) or clinically significant bleeding (33 prescriptions). The INR decreased to 1.5 or less in 40 of 66 prescriptions in this group, but remained prolonged in the other 26 prescriptions. In 16 of 33 bleeding patients, haemostasis was achieved with PTX-VF and other blood products. Some patients had other factors contributing to bleeding that were also treated, including thrombocytopenia (seven patients), concurrent antiplatelet therapy (nine patients), and prolonged APTT (23 patients).

\section{PTX-VF with and without FFP}

The contribution of FFP for warfarin reversal was examined by comparing prescriptions of PTX-VF given with and without FFP (Box 3). A median of two units of FFP was given in the PTX-VF with FFP group and the number of patients prescribed vitamin $K$ was not significantly different between groups $(P=0.15$; odds ratio, 0.49 [ $95 \%$ CI, 0.18 to 1.3$]$ ). There was a significant decrease in INR over time that was independent of group

1 Patient characteristics, PTX-VF treatment details and results of laboratory testing by treatment group

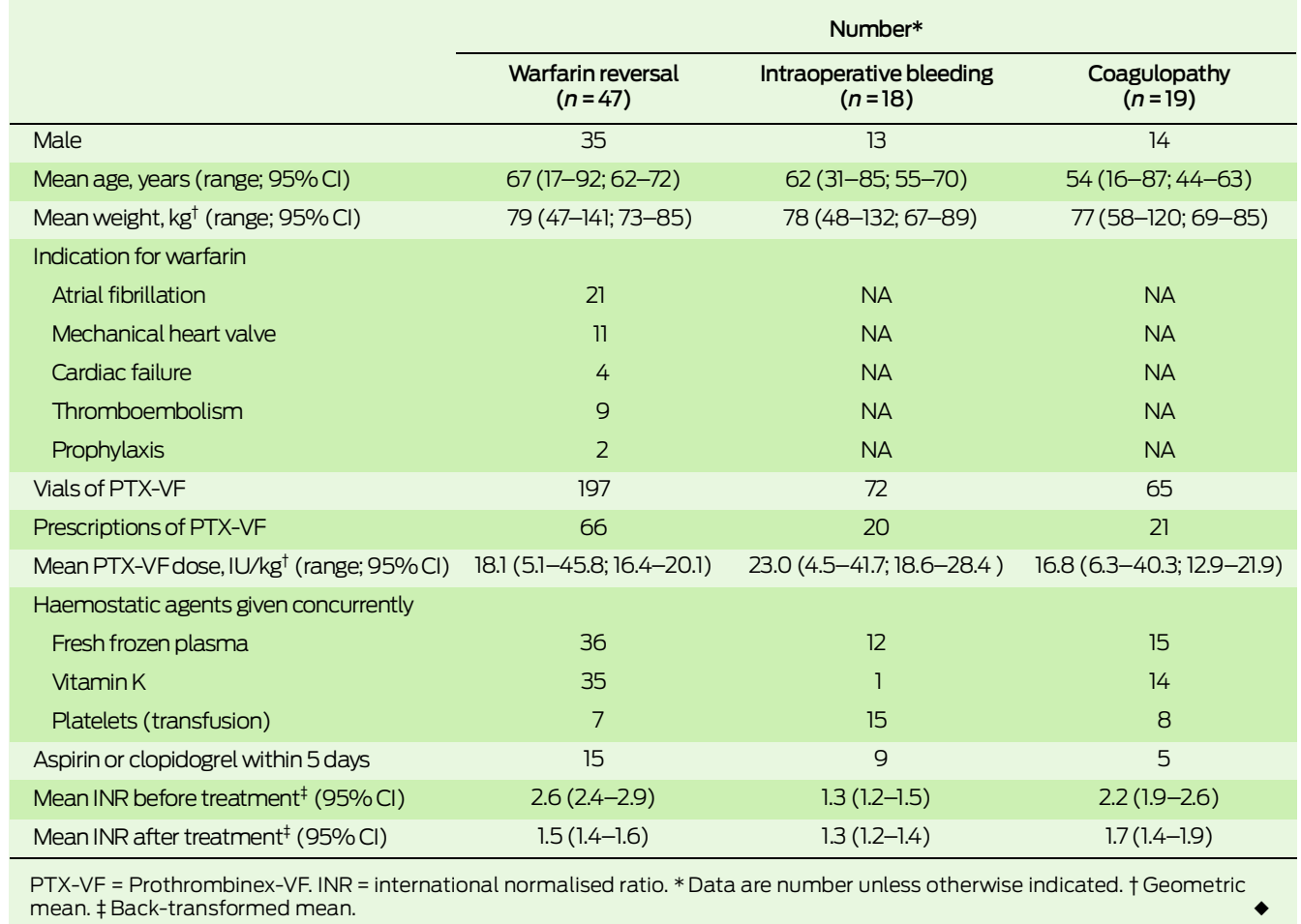

\section{Prothrombinex-VF vials prescribed by specialty and indication}

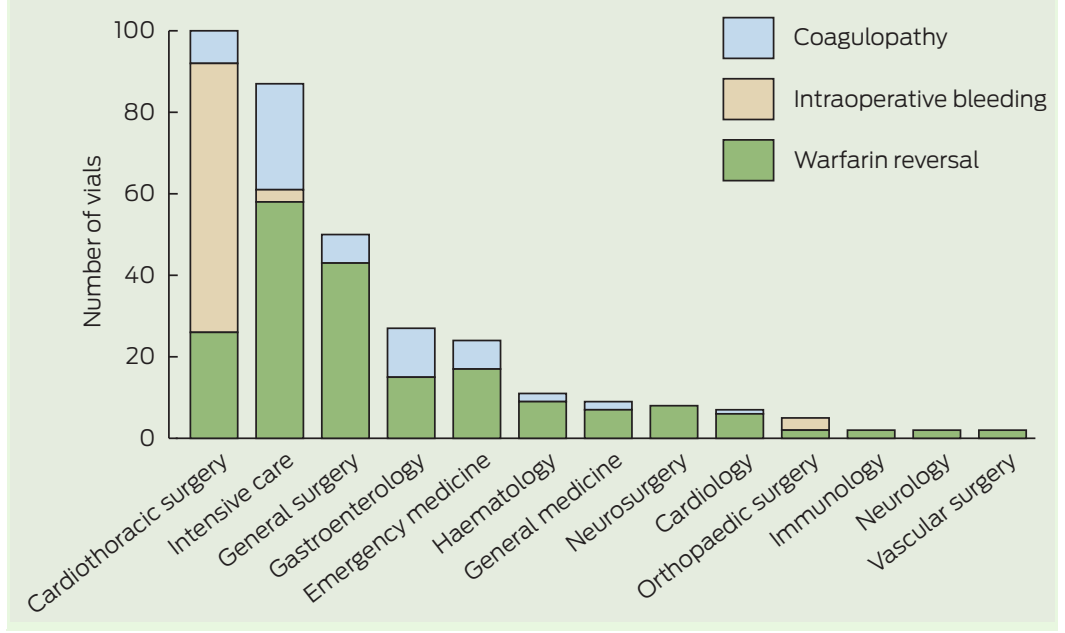

$(P<0.001 ; 95 \% \mathrm{CI}, 0.3$ to 0.4$)$. There was a significant difference in the rate of change in INR between groups $(P=$ $0.008 ; 95 \% \mathrm{CI},-0.2$ to -0.1$)$, with the PTX-VF alone group decreasing by a smaller amount, although this group also had a smaller mean dose of PTXVF $(P=0.007)$.

\section{Lower and higher PTX-VF doses}

Lower doses of PTX-VF (<25 IU/kg) were compared with higher doses (25-50 IU/kg) for warfarin reversal (Box 4). There was a significant difference in mean PTX-VF dose between the groups $(16.1 \mathrm{IU} / \mathrm{kg}$ in lower-dose group $\mathrm{v} 29.1 \mathrm{IU} / \mathrm{kg}$ in higher-dose group; $P<0.001)$. No significant difference was found between groups for the number of FFP units or the number of vitamin $\mathrm{K}$ prescriptions. There was a significant decrease in INR over time that was independent of group $(P=0.002$; $95 \% \mathrm{CI},-3.1$ to -0.7$)$. There was no evidence of a difference in the rate of change in INR between groups ( $P=$ 0.56 ; $95 \% \mathrm{CI},-0.8$ to 1.6 ). 
3 Prescriptions of PTX-VF, with and without fresh frozen plasma, for warfarin reversal

\begin{tabular}{lccc} 
& PTX-VF with FFP $(n=36)$ & PTX-VF alone $(n=30)$ & $P$ \\
\hline Mean PTX-VF dose, IU/kg* $(95 \% \mathrm{Cl})$ & $20.4(18.3-22.9)$ & $15.7(13.2-18.7)$ & 0.007 \\
Mean INR before treatment ${ }^{\dagger}(95 \% \mathrm{Cl})$ & $2.7(2.4-3.2)$ & $2.5(2.2-2.9)$ & - \\
\hline Mean INR after treatment ${ }^{\dagger}(95 \% \mathrm{Cl})$ & $1.4(1.3-1.5)$ & $1.6(1.5-1.8)$ & - \\
Median number of fresh frozen plasma units & 2 & - & - \\
Number of prescriptions given with vitamin K & 22 & 13 & 0.15 \\
Median vitamin K dose, $\mathrm{mg}$ (intravenous) & 10 & 5 & - \\
\hline PTX-VF = Prothrombinex-VF. INR = international normalised ratio. * Geometric mean. $\dagger$ Back-transformed mean.
\end{tabular}

4 Lower-dose versus higher-dose PTX-VF prescriptions for warfarin reversal

\begin{tabular}{lccc} 
& $\begin{array}{c}\text { Lower dose } \\
(<25 I U / \mathrm{kg})(\boldsymbol{n}=52)\end{array}$ & $\begin{array}{c}\text { Higher dose } \\
(25-50 \mathrm{IU} / \mathrm{kg})(n=14)\end{array}$ & $P$ \\
\hline Mean PTX-VF dose, IU/kg* $(95 \% \mathrm{Cl})$ & $16.1(14.4-17.7)$ & $29.1(26.1-32.5)$ & $<0.001$ \\
Mean INR before treatment ${ }^{\dagger}(95 \% \mathrm{Cl})$ & $2.5(2.3-2.9)$ & $2.9(2.3-3.8)$ & - \\
Mean INR after treatment ${ }^{\dagger}(95 \% \mathrm{Cl})$ & $1.5(1.4-1.6)$ & $1.5(1.3-1.8)$ & - \\
Median number of fresh frozen plasma units & 2 & 2 & 0.80 \\
Number of prescriptions given with vitamin $\mathrm{K}$ & 26 & 9 & 0.31 \\
Median vitamin K dose, $\mathrm{mg}$ (intravenous) & 10 & 10 & - \\
\hline
\end{tabular}

PTX-VF = Prothrombinex-VF. INR = international normalised ratio. * Geometric mean. $\dagger$ Back-transformed mean.
PTX-VF (20.0-41.7 IU/kg) during cardiothoracic surgery for haemostasis; five had no complications and the other died from an unrelated cause. Monitoring of factor IX levels was not performed. Fourteen patients died within 7 days of PTX-VF therapy, but these deaths were unrelated to PTXVF (six patients had organ failure, four had sepsis, three had malignancy and one had multiple trauma).

\section{Discussion}

During the 6-month audit period, 334 vials of PTX-VF were used at RPH, which is consistent with average usage; they were used for warfarin reversal and to treat intraoperative bleeding and coagulopathy. No adverse effects from PTX-VF were identified in our audit.

$\mathrm{RPH}$ uses more PTX-VF than any other hospital in WA. Between July 2009 and June 2010, 2911 vials were used statewide, mostly at RPH (747 vials, $26 \%$ of state supply) (unpublished data, Australian Red Cross Blood Service).

Warfarin is commonly used for treatment of atrial fibrillation and thromboembolic disease and for stroke prevention, and the number of patients prescribed warfarin in Australia is expected to increase due to the ageing population. ${ }^{6}$ Safe warfarin reversal is therefore important to permit surgery and treat major bleeding (major bleeding affects about $6.5 \%$ of patients anticoagulated with warfarin annually, and about $1 \%$ of such bleeds are fatal). ${ }^{7}$

We found that PTX-VF is effective for warfarin reversal without FFP, which is consistent with results of previous studies. ${ }^{3,5-7}$ FFP and some fourfactor PCCs ${ }^{1,2,8}$ contain large amounts of factor VII, while PTX-VF and other three-factor PCCs contain little to no factor VII. We therefore believe that factor VII is not important for warfarin reversal, as this can be achieved with PTX-VF and other three-factor PCCs. ${ }^{4-6,9}$ Recent guidelines from the British Committee for Standards in Haematology recommend that FFP should only be used for major bleeding when PCCs are not available. ${ }^{3}$ Advantages of PTX-VF (and other PCCs) over FFP include immediate onset of action, easier storage, easier reconstitution without time needed to thaw, less risk 
of disease transmission, and no risk of transfusion-related lung injury, fluid overload or blood group incompatibility. ${ }^{4-7,9}$ Also, reintroduction of warfarin after surgery to return to a stable anticoagulated state has been successfully shown in patients given PTX-VF. ${ }^{9}$

Lower doses of PTX-VF $(<25 \mathrm{IU} / \mathrm{kg})$ are effective for warfarin reversal. We found significant INR reduction independent of lower-dose or higher-dose group with similar doses of vitamin $\mathrm{K}$ and FFP. Ex-vivo testing of reduction of INR values greater than 4 with one dose (500 IU) of PCC has been found to normalise INR. ${ }^{2}$ However, we cannot recommend that this finding be extrapolated to patients who have an INR value greater than 4 because there were few such patients in our study - most had a pretreatment INR between 2.0 and 3.0.

Adherence to guidelines ${ }^{4}$ was poor in our audit. There were large variations in dose (in IU/kg) and PTX-VF usage in bleeding patients who had normal coagulation profiles before administration of PTX-VF and other blood products. Despite divergence from the warfarin reversal consensus guidelines, the INR reduction to 1.5 or less in most (40 of 66) prescriptions and cessation of bleeding in most cases supports lowering the recommended PTX-VF dose in future revisions of guidelines. When INR did not reduce to 1.5 or less, haemodynamic instability or sepsis was present in most cases.
Use of PCCs in intraoperative bleeding (an indication for which it is not licensed) is emerging. In 2008, a retrospective analysis of the role of PCCs in bleeding surgical patients whose condition had not responded to conventional blood products demonstrated the potential value of PCCs in controlling bleeding during surgery after standard blood products had failed. ${ }^{8}$ In our audit, 66 vials of PTX-VF were prescribed for intraoperative bleeding during cardiothoracic surgery, possibly because of the results of the abovementioned analysis and for its perceived superiority over FFP in thrombin augmentation and subsequent effects on platelet activation. As blood loss was not accurately quantified, plasma factor levels were not checked and the timing of PTX-VF among other blood products varied, conclusions cannot be made about use of PTX-VF in intraoperative bleeding from our data. A prospective study is currently running at $\mathrm{RPH}$ to assess whether PTX-VF significantly decreases blood transfusion requirements in cases of intraoperative bleeding.

When PTX-VF was used to treat coagulopathy in non-warfarinised patients, it was effective at decreasing INR and resulted in haemostasis in nine of 13 bleeding patients. However, major studies of PTX-VF use in such patients (for whom its use is not licensed) are lacking.

Our audit showed that lower doses of PTX-VF $(<25 \mathrm{IU} / \mathrm{kg})$ and PTX-VF without FFP are effective for warfarin reversal. Administering PTX-VF in this manner can simplify warfarin reversal and lead to cost savings and potentially fewer complications. Improved prescribing practices, such as consistency with dosing and measurement of factor IX levels before and after dose, are desirable, and these principles can be applied to other PCCs.

Acknowledgements: We thank Sally Burrows (Biostatistician, School of Medicine and Pharmacology, University of Western Australia and RPH) for performing the data analysis.

Competing interests: No relevant disclosures.

Received 21 Jun 2011, accepted 2 Nov 2011.

1 Junagade P, Grace R, Gover P. Fixed dose prothrombin complex concentrate for the reversal of oral anticoagulation therapy. Hematology 2007; 12: 439-440.

2 Gatt A, Riddell A, van Veen JJ, et al. Optimizing warfarin reversal - an ex vivo study. J Thromb Haemost 2009; 7: 1123-1127.

3 Evans SJ, Biss TT, Wells RH, Hanley JP. Emergency warfarin reversal with prothrombin complex concentrates: UK wide study. Br J Haematol 2008; 141: 268-269.

4 Baker RI, Coughlin PB, Gallus AS, et al; Warfarin Reversal Consensus Group. Warfarin reversal: consensus guidelines, on behalf of the Australasian Society of Thrombosis and Haemostasis. Med J Aust 2004; 181: 492-497.

5 Crawford JH, Augustson BM. Prothrombinex use for the reversal of warfarin: is fresh frozen plasma needed? Med J Aust 2006; 184: 365-366.

6 Tran H, Collecutt M, Whitehead S, Salem HH. Prothrombin complex concentrates used alone in urgent reversal of warfarin anticoagulation. Intern Med J 2011; 41: 337-343.

7 Leissinger CA, Blatt PM, Hoots WK, Ewenstein B. Role of prothrombin complex concentrates in reversing warfarin anticoagulation: a review of the literature. Am J Hematol 2008; 83: 137-143.

8 Bruce D, Nokes TJ. Prothrombin complex concentrate (Beriplex P/N) in severe bleeding: experience in a large tertiary hospital. Crit Care 2008; 12: R105.

9 Chiu D, Grigg M, Levi E. Operating on patients with warfarin: simpler alternative approach. ANZ J Surg 2009; 79: 409-410.
We are seeking entries from Aboriginal or Torres Strait Islander people who are working, researching or training in a health-related field, in two categories:

Essay: up to 2000 words long

Original artwork: Submitted as a digital photograph or video, with a brief description of the health message that the artwork is conveying.

Your entry should present original and positive ideas aimed at promoting health gains and health equity for Australia's Indigenous peoples.

Winning entries will be published in the MJA, and there is a $\$ 2000$ cash prize in each category.

\section{The Dr Ross Ingram Prize 2012}

\section{Now open for submissions of essays and images}

Please see our website for further details, and to be inspired by previous entries.

https://www.mja.com.au/author-centre/ awards/dr-ross-ingram-memorial-prize

Entries can be sent to: rossingramprize@mja.com.au

or

directly to our manuscript submissions site www.editorialmanager.com/mja

Dr Ruth Armstrong - Deputy Editor MJA

Closing date: Friday 4 May 2012 